\title{
A FACTORIZATION THEOREM FOR COMPACT OPERATORS
}

\author{
DANIEL J. RANDTKE
}

\begin{abstract}
It is shown that every compact operator $T: E \rightarrow F$ between Banach spaces admits a compact factorization $(T=Q P$ where $P: E \rightarrow c$ and $Q: c \rightarrow F$ are compact) through a closed subspace $c$ of the Banach space $c_{0}$ of zero-convergent sequences.
\end{abstract}

A (linear) operator $T: E \rightarrow F$ between Banach spaces is compact if $T$ transforms the unit ball of $E$ into a relatively compact subset of $F$. The author has recently shown [3, Corollary 2.10] that an operator $T: E \rightarrow F$ is compact if and only if there is a sequence $\lambda$ in $c_{0}$ and a sequence $\left\{a_{n}\right\}$ in the unit ball of the topological dual $E^{\prime}$ of $E$ such that

for all $x$ in $E$.

$$
\|T x\| \leqq \sup \left|\lambda_{n}\right|\left|\left\langle x, a_{n}\right\rangle\right|
$$

THEOREM. If $T: E \rightarrow F$ is a compact operator between Banach spaces, then there is a closed subspace $c$ of $c_{0}$ and compact operators $P: E \rightarrow c$ and $Q: c \rightarrow F$ with $T=Q P$.

Proof. Suppose that $T: E \rightarrow F$ is compact. Then there is a sequence $\lambda$ in $c_{0}$ and a sequence $\left\{a_{n}\right\}$ in $E^{\prime}$ such that for each $x$ in $E$

$$
\|T x\| \leqq \sup \left|\lambda_{n}\right|^{2}\left|\left\langle x, a_{n}\right\rangle\right| \text {. }
$$

Let $P: E \rightarrow c_{0}$ be the compact operator defined by $P(x)=\left\{\lambda_{n}\left\langle x, a_{n}\right\rangle\right\}$. Let $c$ denote the closure of $P(E)$ in $c_{0}$. Let $D: c \rightarrow c_{0}$ be the compact operator defined by $D(\xi)=\left\{\lambda_{n} \xi_{n}\right\}$. Let $S: D(c) \rightarrow F$ be the (unique) bounded (by (*), $\|S\| \leqq 1)$ operator such that $S(D P x)=T(x)$ for all $x$ in $E$. Let $Q=S D$. Then $T=Q P$, where both $Q$ and $P$ are compact.

REMARK 1. Grothendieck [1, Chapitre I, p. 165] has shown that a Banach space $E$ has the approximation property if, for every Banach space $F$ and every compact operator $T: F \rightarrow E$, there exists a sequence of finite rank operators $T_{n}: F \rightarrow E$ with $\left\|T_{n}-T\right\| \rightarrow 0$. This result together with our factorization theorem can be used to give an elementary proof

Received by the editors August 3, 1971 and, in revised form, November 2, 1971. AMS 1970 subject classifications. Primary 47B05; Secondary 46B99.

Key words and phrases. Banach space, compact operator, approximation property, Hilbert space. 
of the following result of Grothendieck [1, Chapitre I, pp. 170-171]: If each closed subspace $c$ of $c_{0}$ has the approximation property, then every Banach space has the approximation property.

REMARK 2. Lindenstrauss and Tzafriri [2, p. 265] have recently shown that a Banach space $E$ is isomorphic to a Hilbert space if and only if, for every closed subspace $F$ of $E$, every compact operator $T: F \rightarrow F$ can be extended to a bounded operator $S: E \rightarrow F$. By combining this result with our factorization theorem it follows that a Banach space $E$ is isomorphic to a Hilbert space if and only if, for every closed subspace $F$ of $E$ and every closed subspace $c$ of $c_{0}$, every compact operator $T: F \rightarrow c$ can be extended to a bounded operator $S: E \rightarrow c$. This result contrasts with the following (unpublished) result of the author: If $E$ is an infinite dimensional Hilbert space, then there exists a closed subspace $c$ of $c_{0}$ and a compact operator $T: c \rightarrow E$ that cannot be extended to a bounded operator from $c_{0}$ into $E$.

The author would like to thank the referee for his comments and references.

\section{REFERENCES}

1. A. Grothendieck, Produits tensoriels topologiques et espaces nucléaires, Mem. Amer. Math. Soc. No. 16 (1955). MR 17, 763.

2. J. Lindenstrauss and L. Tzafriri, On the complemented subspaces problem, Israel. J. Math. 9 (1971), 263-269.

3. D. J. Randtke, Characterizations of precompact maps, Schwartz spaces and nuclear spaces, Trans. Amer. Math. Soc. 165 (1972), 87-101.

Department of Mathematics, University of Georgia, Athens, Georgia 30601 